\title{
Contracting Few Edges to Remove Forbidden Induced Subgraphs
}

\author{
Leizhen Cai ${ }^{\star}$ and Chengwei Guo \\ Department of Computer Science and Engineering, \\ The Chinese University of Hong Kong, Hong Kong S.A.R., China
}

$\{$ Icai, cwguo\}@cse.cuhk.edu.hk

\begin{abstract}
For a given graph property $\Pi$ (i.e., a collection $\Pi$ of graphs), the $\Pi$-CONTRACTION problem is to determine whether the input graph $G$ can be transformed into a graph satisfying property $\Pi$ by contracting at most $k$ edges, where $k$ is a parameter. In this paper, we mainly focus on the parameterized complexity of $\Pi$-CONTRACTION problems for $\Pi$ being $H$-free (i.e., containing no induced subgraph isomorphic to $H$ ) for various fixed graphs $H$.

We show that Clique Contraction (equivalently, $P_{3}$-Free ConTRACTION for connected graphs) is FPT (fixed-parameter tractable) but admits no polynomial kernel unless $N P \subseteq \operatorname{coNP} /$ poly, and prove that Chordal Contraction (equivalently, $\left\{C_{l}: l \geq 4\right\}$-Free ContraCTION) is W[2]-hard. We completely characterize the parameterized complexity of $H$-Free Contraction for all fixed 3-connected graphs $H$ : FPT but no polynomial kernel unless $N P \subseteq \operatorname{coNP} /$ poly if $H$ is a complete graph, and $\mathrm{W}[2]$-hard otherwise. We also show that $H$-FreE CONTRACTION is W[2]-hard whenever $H$ is a fixed cycle $C_{l}$ for some $l \geq 4$ or a fixed path $P_{l}$ for some odd $l \geq 5$.
\end{abstract}

\section{Introduction}

Edge contraction is a fundamental operation in graph theory, and plays a crucial role in the celebrated graph minor theory. An edge contraction in a graph identifies two endpoints of an edge, and eliminates loop and multiple edges in the resulting graph. For a given graph property $\Pi$ (i.e., a collection $\Pi$ of graphs), the $\Pi$-CONTRACTION problem asks whether the input graph can be modified into a $\Pi$-graph, i.e. a graph satisfying property $\Pi$, by at most $k$ edge contractions.

The complexity of edge contraction problems has been studied in the literature, but does not receive as much attention as graph modification problems in terms of vertex and edge addition/deletion. Watanabe et al. [15] and Asano and Hirata [1,2] proved that $\Pi$-CONTRACTION is NP-complete if $\Pi$ is finitely characterized by 3 -connected forbidden subgraphs, or $\Pi$ is hereditary on contractions and is determined by biconnected components.

\footnotetext{
* Partially supported by GRF grant CUHK410409 of the Research Grants Council of Hong Kong.
}

G. Gutin and S. Szeider (Eds.): IPEC 2013, LNCS 8246, pp. 97-109 2013.

(C) Springer International Publishing Switzerland 2013 
Recently, researchers have studied edge contraction problems from the perspective of parameterized complexity. Heggernes et al. [1] have obtained an FPT algorithm for BIPARTITE CONTRACTION that asks whether a graph can be modified into a bipartite graph by at most $k$ edge contractions. Later Heggernes et al. 10 presented a $4.98^{k} n^{O(1)}$ time algorithm for TREE CONTRACTION and a $2^{k+o(k)}+n^{O(1)}$ time algorithm for PATH Contraction. Golovach et al. [7] considered $\Pi$-CONTRACTION for $\Pi$ being the class of graphs of minimum degree at least $d$ and showed that the problem is FPT when both $d$ and $k$ are parameters, but W[1]-hard when only $k$ is the parameter and NP-complete when $d=14$. Furthermore, Golovach et al. 8 showed that Planar Contraction is FPT.

In this paper, we focus on the parameterized complexity of the following $H$-Free Contraction problems, where a graph is $H$-free if it contains no induced copy of $H$, i.e., an induced subgraph isomorphic to $H$. We note that several important graph classes (e.g., cographs, triangle-free graphs, and clawfree graphs) are characterized by $H$-freeness.

\section{H-Free Contraction \\ Instance: Graph $G$, positive integer $k$ as parameter. \\ Question: Can we obtain an $H$-free graph from $G$ by at most $k$ edge contractions?}

It is easy to see that whenever $H$ is a fixed complete graph $K_{t}, H$-FreE Contraction is FPT as the only way to destroy a copy of $K_{t}$ is to contract some edges in the copy, which implies an FPT algorithm by the bounded search tree method. However, the situation for $H$ other than complete graphs is very complicated as contractions can occur for edges not involved in any induced copies of $H$. In this paper, we try to determine the parameterized complexity of $H$-Free Contraction in terms of the structure of $H$, and we have made important progress towards this goal by the following results:

- Clique Contraction (equivalently, $P_{3}$-Free Contraction for connected graphs) is FPT but admits no polynomial kernel unless $N P \subseteq \operatorname{coNP} /$ poly, and $P_{l}$-Free Contraction is W[2]-hard for every fixed path $P_{l}$ with odd $l \geq 5$.

- $C_{3}$-Free Contraction is FPT but admits no polynomial kernel unless $N P \subseteq \operatorname{coNP} /$ poly, and $C_{l}$-Free Contraction is W[2]-hard for every fixed cycle $C_{l}$ with $l \geq 4$.

- Chordal Contraction is W[2]-hard, which is in contrast to that both Chordal Completion and Chordal Deletion are FPT [3, 12, 13.

- For every fixed 3-connected graph $H, H$-Free Contraction is W[2]-hard whenever $H$ is not a complete graph. Otherwise, it is FPT but admits no polynomial kernel unless $N P \subseteq \operatorname{coNP} /$ poly.

Our FPT algorithm for Clique Contraction first finds a large "seed clique" in the input graph, and then uses a branch-and-search algorithm to contract other edges into the clique. This idea is useful for other edge contraction problems such as Split Contraction, which will appear in our future paper. For 
the W[2]-hardness proofs, all FPT reductions in this paper are from the classical Dominating Set problem that takes an integer $k$ as parameter, and asks whether an input graph $G$ contains a dominating $k$-set, i.e., at most $k$ vertices $V^{\prime}$ s.t. every vertex in $V(G)-V^{\prime}$ is adjacent to some vertex in $V^{\prime}$.

All graphs in the paper are simple, finite, and undirected. For a graph $G$, we denote its vertex set and edge set by $V(G)$ and $E(G)$ respectively. A graph is chordal if it has no induced cycle of size greater than 3. For an integer $t, K_{t}$ is a complete graph on $t$ vertices, $C_{t}$ is a cycle on $t$ vertices, and $P_{t}$ is a path on $t$ vertices. The contraction of edge $u v$ in $G$ removes $u$ and $v$ from $G$, and replaces them by a new vertex adjacent to precisely those vertices that were adjacent to at least one of $u$ or $v$. For a set of edges $F \subseteq E(G)$, we use $G / F$ to denote the graph obtained from $G$ by sequentially contracting all edges in $F$. If a graph $H$ with vertex set $\left\{h_{1}, \cdots, h_{l}\right\}$ can be obtained from graph $G$ by a sequence of edge contractions, then $G$ is contractible to $H$. In this case, $G$ has a $H$-witness structure: a partition of $V(G)$ into $l$ sets $W\left(h_{1}\right), \cdots, W\left(h_{l}\right)$, called witness sets, such that each $W\left(h_{i}\right)$ induces a connected subgraph of $G$ and for any two $W\left(h_{i}\right)$ and $W\left(h_{j}\right)$, there is an edge between $W\left(h_{i}\right)$ and $W\left(h_{j}\right)$ in $G$ iff $h_{i} h_{j} \in E(H)$. We obtain $H$ from $G$ by contracting vertices in each $W\left(h_{i}\right)$ into a single vertex.

\section{Path-Free Contraction}

We start with $P_{l}$-Free Contraction problems for fixed $l \geq 3$. Since edge contractions preserve the connectedness of a graph and a graph is a complete graph iff it is $P_{3}$-free and connected, $P_{3}$-FreE Contraction for connected graphs is equivalent to Clique Contraction that asks whether we can transform the input graph into a clique (i.e., complete graph) by contracting at most $k$ edges.

We note that transforming a $n$-vertex graph $G$ into a clique by contracting $k$ edges is equivalent to finding a $(n-k)$-clique minor of $G$ as an edge contraction reduces the number of vertices by one. Thus Clique Contraction is a parametric dual of MAXIMUM CLIQUE Minor that takes as input a graph $G$ and an integer $h$, and asks whether $G$ contains a clique $K_{h}$ as a minor. MAXImum Clique Minor is NP-complete as shown by Eppstein [6], and FPT when parameterized by $h$ following a celebrated result on graph minors by Robertson and Seymour [14. The NP-completeness of Clique Contraction directly follows from that of MAXimum Clique Minor. Here we present an FPT algorithm for Clique Contraction, which combines bounded search tree with a kernelization of the problem from the second author's $\mathrm{PhD}$ dissertation 9 .

Theorem 1. Clique Contraction can be solved in $O\left(2^{7 k} k^{2 k+5}+m\right)$ time, but admits no polynomial kernel unless $N P \subseteq \operatorname{coNP} /$ poly.

Proof. For a vertex set $A$, we denote by $E[A]$ the set of edges whose both endpoints are in $A$. For any two disjoint vertex sets $B$ and $C$, we use $E[B, C]$ to denote the set of edges whose one endpoint is in $B$ and the other is in $C$.

Since each edge contraction affects only two vertices, a $n$-vertex graph $G$ must contain a clique of $(n-2 k)$ vertices $V_{c}$ in order for $G$ to be contractible 
to a clique by at most $k$ contractions. We start by using an FPT algorithm for VERTEX COver to find such vertex set $V_{c}$. Next, we construct a bounded search tree and consider all possible edges in the solution set. In the search tree, we branch out by contracting edges of $E\left(G-V_{c}\right)$, edges of $E\left[V_{c}, V(G)-V_{c}\right]$, and edges of $E\left[V_{c}\right]$ in sequence. See Fig. 1 for an illustration. Note that the number of edges in $E\left[V_{c}, V(G)-V_{c}\right]$ or $E\left[V_{c}\right]$ might be very large. However, we do not need to consider all edges. The trick is to compress the possible choices into a special set of edges whose size is bounded by a function of $k$. Our algorithm consists of the following steps:

1. Determine whether there is a set $V_{c}$ of $n-2 k$ vertices that induces a clique in $G$. If yes, find $V_{c}$ and let $V_{k}=V(G)-V_{c}$; otherwise, return "NO".

2. We construct a search tree and label the root by the input instance $(G, k)$. We branch out at the root by contracting every possible set of at most $k$ edges in $E\left[V_{k}\right]$ and label the new node of the tree by the resulting instance $\left(G^{\prime}, k^{\prime}\right)$, where $k^{\prime}$ is the number of remaining edge contractions.

3. For each node $\left(G^{\prime}, k^{\prime}\right)$ obtained in Step 2, we assume that vertices of $V_{k}$ are contracted into vertices $V_{k}^{\prime}$ in $G^{\prime}$. We branch out by every possible partition $V_{k}^{\prime}=\left(V_{p}, R\right)\left(V_{p}\right.$ corresponds to the subset of $V_{k}^{\prime}$ consisting of vertices not involved in edge contractions). Let $T=\left\{v \in V_{c} \mid \exists w \in V_{p}, w v \notin E\left(G^{\prime}\right)\right\}$. If $|R|>k^{\prime}$ or $|T|>2 k^{\prime}$, discard this node.

We continue to branch by contracting every possible set of $|R|$ edges in $E[R, T]$ that covers all vertices in $R$ and label the new node of the tree by the resulting instance $\left(G^{\prime \prime}, k^{\prime \prime}\right)$. Here $k^{\prime \prime}=k^{\prime}-|R|$, and vertices in $R$ are merged into the large clique $G^{\prime \prime}\left[V_{c}\right]$.

4. For each node $\left(G^{\prime \prime}, k^{\prime \prime}\right)$ obtained in Step 3 , we arbitrarily choose a vertex $u \in V_{c}-T$. We branch by contracting every $k^{\prime \prime}$-subset of $E[T \cup\{u\}]$.

5. If there exists a leaf in this search tree labelled with a clique (in Step 4), then return "YES"; otherwise, return "NO".

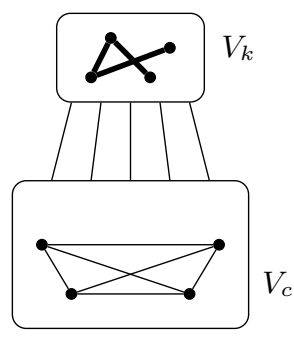

Step 2

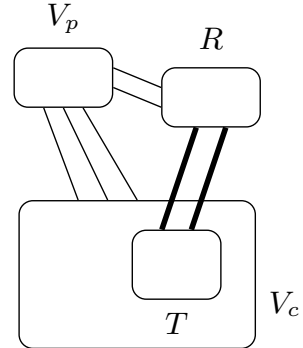

Step 3

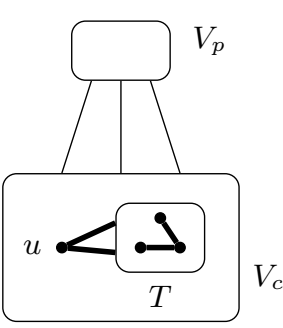

Step 4

Fig. 1. Edges being considered for contractions (thick edges) in Step 2-4 
In Step 1, finding a $(n-2 k)$-clique is equivalent to finding a $2 k$-vertex cover in the complement graph of $G$, which costs $O\left(1.2738^{2 k}+k n\right)$ time following a known algorithm by Chen et. al. [4]. In Step 2, the root has at most $\sum_{k^{\prime}}\left(\begin{array}{c}\left(\begin{array}{c}V_{k} \mid \\ 2 \\ k-k^{\prime}\end{array}\right) \\ k\end{array}\right) \leq$ $\sum_{k^{\prime}}\left(2 k^{2}\right)^{k-k^{\prime}}$ children. In Step 3, the total number of different partitions $V_{k}^{\prime}=$ $\left(V_{p}, R\right)$ is $2^{\left|V_{k}^{\prime}\right|} \leq 2^{2 k}$, and for each partition we branch into at most $|R||T| \leq 2 k^{\prime 2}$ nodes. In Step 4 , for each node we branch into at most $\left(\left(\begin{array}{c}|T|+1 \\ 2\end{array}\right)\right)^{k^{\prime \prime}} \leq\left(2 k^{2}\right)^{k^{\prime}}$ leaves. Therefore the size of this search tree is bounded by $\sum_{k^{\prime}}\left(2 k^{2}\right)^{k-k^{\prime}} 2^{2 k} 2 k^{\prime 2}\left(2 k^{2}\right)^{k^{\prime}}=$ $O\left(2^{3 k} k^{2 k+3}\right)$, and each node of the tree takes $O(m)$ time to generate. Thus, the total running time of our branching algorithm is $O\left(1.2738^{2 k}+k n\right)+O\left(2^{3 k} k^{2 k+3}\right)$ $O(m)=O\left(2^{3 k} k^{2 k+3} m\right)$. Following a general result in the second author's $\mathrm{PhD}$ dissertation [9], Clique ContraCtion has a kernel of $O\left(2^{2 k} k\right)$ vertices, which can be constructed in linear time. Combining this exponential kernel with our branching algorithm, we obtain an FPT algorithm running in time $O\left(2^{7 k} k^{2 k+5}+m\right)$.

For the correctness of the algorithm, it is easy to see that $(G, k)$ has a solution when our algorithm outputs "YES". On the other hand, suppose that $G$ contains a solution set $S$ of size $k$. Our branching algorithm indeed simulates the procedure of contracting $S$ in $G$. First after contracting edges $S \cap E\left[V_{k}\right]$, vertex set $V_{k}$ is modified into a set $V_{k}^{\prime}=V_{p} \cup R$ where $V_{p}$ consists of vertices that are not involved in any edges of $S \backslash E\left[V_{k}\right]$. Note that $T$ is the set of vertices in $V_{c}$ that are not adjacent to at least one vertex of $V_{p}$. To make $G$ into a clique, every vertex in $T$ must be incident with some edge in $S \backslash E\left[V_{k}\right]$, implying that $T \leq 2 k^{\prime}$ where $k^{\prime}=\left|S \backslash E\left[V_{k}\right]\right|$. For an arbitrary vertex $u \in V_{c}-T$, we construct an edge set $S^{*}$ from $S$ by removing edges $\left\{x y \in S: x, y \in V_{c}-T\right\}$ and replacing every $x y$ in $S$ with $x \in T$ and $y \in V_{c}-T$ by $x u$. It can be shown that $S^{*}$ is also a solution of $(G, k)$, and by Step 4 there always be a leaf in the search tree labelled with $G / S^{*}$, implying that the algorithm outputs "YES". The complete proof will be given in the full paper.

We now turn to the non-existence of polynomial kernels for Clique ConTRACTION. Due to space limit, we will only sketch the main idea here and give the complete proof in the full paper. First we show that the following One-Sided Dominating Set problem admits no polynomial kernel unless $N P \subseteq \operatorname{coNP} /$ poly: Given a bipartite graph $G=(X, Y ; E)$ and an integer $t$ with $|X|$ being the parameter, does $X$ have a subset of at most $t$ vertices that dominates $Y$ ? The NP-completeness of the unparameterized version of the problem easily follows from that of Dominating SET, and we can show that ONE-Sided Dominating SET is OR-compositional, implying that it admits no polynomial kernel unless $N P \subseteq c o N P /$ poly. Note that this problem is different from REDBlue Dominating Set (defined by Dom et al. [5]) whose solution set is in $Y$ instead of $X$.

Next we give a polynomial parameter transformation from ONE-SIDED DoMinating Set to Clique Contraction. The main idea of the transformation is as follows: First we construct a bipartite graph $G^{\prime}=\left(X^{\prime}, Y^{\prime} ; E^{\prime}\right)$ from $G=(X, Y ; E)$ by adding $|X|-t$ new vertices $Z$ to $X$ and make them adjacent to every vertex of $Y$, and adding a new vertex $w$ to $Y$ and make it adjacent to 
every vertex of $X$ (see Fig. 2). Note that each vertex in $Z$ must combine with some vertices in $X$ to form a dominating set for $Y^{\prime}=Y \cup\{w\}$. It is easy to see that $X$ has a dominating $t$-set for $Y$ iff $X^{\prime}$ can be partitioned into $|X|-t+1$ disjoint dominating sets for $Y^{\prime}$.

Then we replace $Y^{\prime}$ by $2\left(\left|X^{\prime}\right|-t^{\prime}\right)+1=2|X|-1$ copies $Y_{1}, \cdots, Y_{2|X|-1}$ of $Y$ where $t^{\prime}=|X|-t+1$, connect every $a \in Y_{i}$ to $b \in X^{\prime}$ iff $a b \in E^{\prime}$ for $i=1, \cdots, 2|X|-1$, and make $X^{\prime}$ and $Y_{1} \cup \cdots \cup Y_{2|X|-1}$, respectively, into two cliques to form graph $G^{\prime \prime}$ (see Fig. 2). If $X^{\prime}$ can be partitioned into $t^{\prime}$ disjoint sets $S_{1}, \cdots, S_{t^{\prime}}$ each of which dominates $Y^{\prime}$, then we can contract vertices in each $S_{i}$ into a single vertex to make $G^{\prime \prime}$ into a clique. The total number of edge contractions we use is $\Sigma_{i}\left(\left|S_{i}\right|-1\right)=\left(\Sigma_{i}\left|S_{i}\right|\right)-t^{\prime}=\left|X^{\prime}\right|-t^{\prime}$. Conversely if $G^{\prime \prime}$ contains $\left|X^{\prime}\right|-t^{\prime}$ edges whose contractions yield a clique, then obviously there exists some $Y_{j}$ whose vertices are not involved in edge contractions. It is easy to see that vertices in each witness set of $X^{\prime}$ form a dominating set for $Y_{j}$ and the number of different witness sets in $X^{\prime}$ is at least $t^{\prime}$, implying that $X^{\prime}$ can be partitioned into $t^{\prime}$ disjoint dominating sets for $Y^{\prime}$.

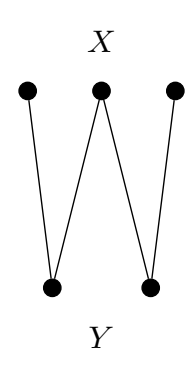

$G$

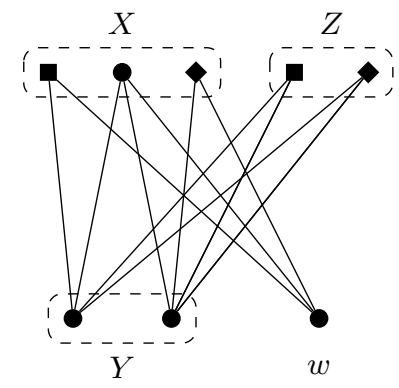

$G^{\prime}$

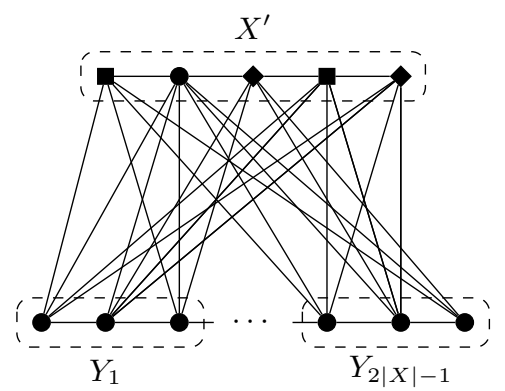

$G^{\prime \prime}$

Fig. 2. An example of the transformation from One-Sided Dominating Set to Clique Contraction with $t=1$

Since $X$ has a dominating $t$-set for $Y$ in $G$ iff $G^{\prime \prime}$ can be modified into a clique by using $\left|X^{\prime}\right|-t^{\prime}=|X|-1$ edge contractions, Clique Contraction admits no polynomial kernel unless $N P \subseteq \operatorname{coNP} /$ poly.

Because $P_{3}$-Free Contraction on connected graphs is equivalent to CLIQUe Contraction, we immediately have the following result.

Corollary 2. $P_{3}$-Free ContraCtion is FPT but admits no polynomial kernel unless $N P \subseteq$ coNP/poly.

On the other hand, $P_{l}$-Free Contraction is hard for every odd $l \geq 5$.

Theorem 3. For every fixed odd $l \geq 5, P_{l}$-Free Contraction is W[2]-hard.

Proof. First we note the following easy FPT reduction from $P_{l}$-FreE ConTRACTiOn to $P_{l+2}$-Free Contraction for every $l \geq 3$ : For any graph $G$ and positive 
integer $k$, we construct a graph $G^{\prime}$ by attaching $k+1$ leaves to each vertex $v$ of $G$, i.e., adding $k+1$ new vertices and connecting them to $v$ with new edges. It is easy to see that $(G, k)$ is a yes-instance of $P_{l}$-Free Contraction iff $\left(G^{\prime}, k\right)$ is a yes-instance of $P_{l+2}$-FreE CONTRACTION.

Therefore we need only prove the theorem for the base case $l=5$. For this purpose, we give an FPT reduction from Dominating SET to $P_{5}$-Free Contraction.

Given an instance $(G, k)$ with $V(G)=\left\{v_{1}, \cdots, v_{n}\right\}$, we construct in polynomial time a graph $G^{\prime}$ as follows (see Fig. 3 for an illustration):

- Create an independent set $\left\{x_{1}, \cdots, x_{n}\right\}$ and a clique $\left\{y_{1}, \cdots, y_{n}\right\}$.

- Make $x_{i}$ adjacent to $y_{j}$ iff $i=j$ or $v_{i} v_{j} \in E(G)$.

- Create a new vertex $u$ and make it adjacent to every vertex of $\left\{y_{1}, \cdots, y_{n}\right\}$.

- Create a $(k+1)$-clique $\left\{z_{1}, \cdots, z_{k+1}\right\}$, where each $z_{i}$ is made adjacent to $u$ and has a new vertex $w_{i}$ attaching to it.

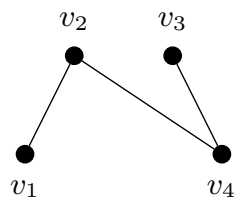

$(a)$

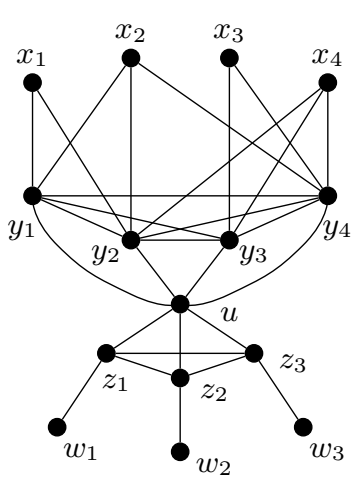

(b)

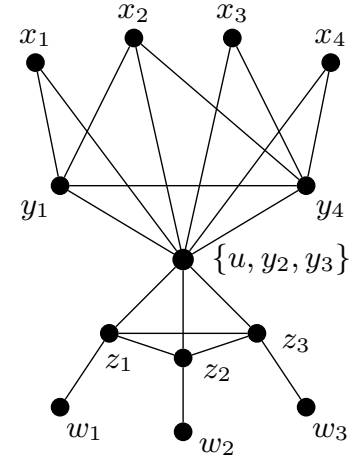

(c)

Fig. 3. (a) Graph $G$ with dominating set $\left\{v_{2}, v_{3}\right\}$; (b) Graph $G^{\prime}$ obtained from $G$; (c) $P_{5}$-free graph $G^{*}$ obtained from $G^{\prime}$ by contracting $\left\{u y_{2}, u y_{3}\right\}$

We claim that $G$ has a dominating $k$-set iff $G^{\prime}$ can be made into $P_{5}$-free by contracting at most $k$ edges.

Suppose that $T$ is a dominating $k$-set in $G$. We contract $k$ edges $\left\{u y_{i}: v_{i} \in T\right\}$ in $G^{\prime}$ to obtain a graph $G^{*}$. Note that $u$ is made adjacent to every vertex of $\left\{x_{1}, \cdots, x_{n}\right\}$ in $G^{*}$. It is easy to see that $G^{*}$ contains no induced 5-path.

Conversely, suppose that $G^{\prime}$ contains at most $k$ edges $F$ whose contraction results in a $P_{5}$-free graph. We show that there exists a dominating $k$-set in $G$. We may assume $k<n$, otherwise $G$ always has a dominating $k$-set. Observe that at least one induced path $\left(u, z_{r}, w_{r}\right)$ for some $1 \leq r \leq k+1$ survives after contracting $F$, which implies that each induced 3-path from some $x_{i}$ to $u$ must be destroyed to make $G^{\prime} P_{5}$-free. Thus distance $d_{G^{\prime} / F}\left(u, x_{i}\right) \leq 1$ for $i=1, \cdots, n$.

We now use $F$ to obtain a dominating set of $G$. Let $R=\left\{x_{i}: \exists v, v x_{i} \in F\right\}$ and $R^{*}=\left\{y_{i}: x_{i} \in R\right\}$. Let $S$ be a set of vertices in $\left\{y_{1}, \cdots, y_{n}\right\}$ that are 
finally in the same witness set with $u$ in the graph $G^{\prime} / F$. It is easy to see that $\left|R^{*}\right|+|S|=|R|+|S| \leq|F| \leq k$. Since for each $1 \leq i \leq n, d_{G^{\prime} / F}\left(u, x_{i}\right) \leq 1$, vertex $x_{i}$ is either contained in $R$ or adjacent to some vertex of $S$ in $G^{\prime}$. This implies that $R^{*} \cup S$ dominates $\left\{x_{1}, \cdots, x_{n}\right\}$, and thus $G$ has a dominating set of at most $k$ vertices.

The reduction in Theorem 3 does not work for even number $l$, and new ideas are needed to deal with even $l$.

\section{Cycle-Free Contraction}

In this section, we consider contraction problems concerning cycles. We show that $C_{l}$-Free Contraction is $\mathrm{W}[2]$-hard for every fixed $l \geq 4$, and the reduction in our proof also implies that CHORdal ContraCtion, which is the same as $\left\{C_{l}: l \geq 4\right\}$-Free Contraction, is also W[2]-hard. It is worth noting that two related graph modification problems CHORDAL COMPLETION and CHORDAL DELETION are both FPT 312.13, which gives us some evidence that contraction seems harder than edge and vertex addition/deletion.

Theorem 4. $C_{l}$-Free Contraction is FPT for $l=3$, but W[2]-hard for every fixed $l \geq 4$.

Proof. For $l=3$, the problem is the same as $K_{3}$-Free Contraction which can be easily solved in $O\left(3^{k} n^{3}\right)$ time using bounded search tree.

For every fixed $l \geq 4$, we provide an FPT reduction from Dominating SeT to $C_{l}$-Free Contraction.

Given a graph $G$ with $V(G)=\left\{v_{1}, \cdots, v_{n}\right\}$, we construct in polynomial time a graph $G^{\prime}$ as follows:

- Create an independent set $\left\{x_{1}, \cdots, x_{n}\right\}$ and a clique $\left\{y_{1}, \cdots, y_{n}\right\}$.

- Make $x_{i}$ adjacent to $y_{j}$ iff $i=j$ or $v_{i} v_{j} \in E(G)$.

- Create a new vertex $u$ and make it adjacent to every vertex of $\left\{y_{1}, \cdots, y_{n}\right\}$.

- For each $x_{i}$, create a length-2 path and a length- $(l-2)$ path whose two ends are identified with $u$ and $x_{i}$, these two paths form an induced $l$-cycle $H_{i}$.

For convenience, we refer to these $n$ induced $l$-cycles $H_{1}, \cdots, H_{n}$ as $u$-cycles. We claim that $G$ has a dominating $k$-set iff $G^{\prime}$ can be made into a $C_{l}$-free graph by contracting at most $k$ edges.

Suppose that $T$ is a dominating $k$-set in $G$, we contract $k$ edges $\left\{u y_{i}: v_{i} \in\right.$ $T\}$ in $G^{\prime}$. In the resulting graph $G^{*}, u$ is made adjacent to all vertices of $\left\{x_{1}, \cdots, x_{n}\right\}$. Therefore all $u$-cycles are destroyed and the size of the largest induced cycle in $G^{*}$ is $l-1$, implying that $G^{\prime}$ is $C_{l}$-free.

Conversely, suppose that $G^{\prime}$ contains at most $k$ edges $F$ whose contraction results in a $C_{l}$-free graph. In particular, all $u$-cycles are destroyed in $G^{\prime} / F$. We may assume $k<n$, otherwise $G$ always has a dominating $k$-set.

We consider the intersection between $F$ and $u$-cycles. Let $F_{i}=F \cap E\left(H_{i}\right)$ for $i=1, \cdots, n$. Observe that the only $u$-cycle destroyed by contraction of $F_{i}$ is 
$H_{i}$, which can also be destroyed by contracting $x_{i} y_{i}$. Thus for every $F_{i}$ that is non-empty, we replace $F_{i}$ by a single edge $\left\{x_{i} y_{i}\right\}$, and then obtain a set $F^{*}$ from $F$ whose contraction destroys all $u$-cycles in $G^{\prime}$. Since none edge of $F^{*}$ lies in any $u$-cycle, then for each $1 \leq i \leq n, u$ is either made adjacent to $x_{i}$ or identified with $x_{i}$ by contracting $F^{*}$, i.e., $d_{G^{\prime} / F^{*}}\left(u, x_{i}\right) \leq 1$. Using the same argument in Theorem 33, we can use $F^{*}$ to obtain a dominating set of $G$ containing at most $\left|F^{*}\right| \leq k$ vertices.

Our proof in Theorem 4 actually shows that $\Pi$-CONTRACTION is W[2]-hard for $\Pi$ being the class of graphs without induced cycles of length $\geq l$ for any fixed $l \geq 4$. We note that for $l=3, \Pi$ coincides with forests, and the problem becomes FPT as shown by Heggernes et al. [10. For $l=4, \Pi$ is exactly the class of chordal graphs, and thus we have the following theorem for CHORDAL CONTRACTION.

Theorem 5. ChORdal Contraction is W[2]-hard.

\section{$4 \quad H$-Free for 3 -Connected $H$}

Asano and Hirata 1 showed that $\Pi$-ConTRACTION is NP-complete whenever $\Pi$ is characterized by a finite forbidden set of 3-connected graphs. However, their reduction is not an FPT reduction and not useful in dealing with the parameterized complexity of $H$-FreE CONTRACTION. In this section, we fully characterize the parameterized complexity of $H$-FreE CONTRACTION for 3-connected $H$.

Theorem 6. Let $H$ be a fixed 3-connected graph. If $H$ is a complete graph, then H-Free Contraction is FPT but admits no polynomial kernel unless $N P \subseteq c o N P /$ poly. Otherwise H-Free Contraction is W[2]-hard.

Proof. If $H$ is a complete graph $K_{t}$ with $t \geq 3$, we can easily obtain an FPT algorithm running in $O\left(\left(\begin{array}{c}t \\ 2\end{array}\right)^{k} n^{t}\right)$ time by bounded search tree as the only way to destroy a copy of $K_{t}$ is to contract some edges in the copy. To show that the problem has no polynomial kernel, we introduce a constrained satisfiability problem RESTRICTED-1S-IN-4 SAT, prove that it is NP-complete and ORcompositional and thus admits no polynomial kernel unless $N P \subseteq \operatorname{coNP} /$ poly, and then give a polynomial parameter transformation from it to our problem $K_{t}$-Free Contraction. Due to space limit, we omit the lengthy proofs here, which are available from the $\mathrm{PhD}$ dissertation (§5.2) of the second author 9.

For the W[2]-hardness part of the theorem, we consider two cases in terms of the structure of $H$.

Case 1. $H$ is not chordal. We give an FPT reduction from Dominating SET to $H$-Free Contraction. For a graph $G$ with $V(G)=\left\{v_{1}, \cdots, v_{n}\right\}$, we construct a graph $G^{\prime}$ as follows (see Fig. 4 for an illustration):

- Create an independent set $\left\{x_{1}, \cdots, x_{n}\right\}$ and a clique $\left\{y_{1}, \cdots, y_{n}\right\}$.

- Make $x_{i}$ adjacent to $y_{j}$ iff $i=j$ or $v_{i} v_{j} \in E(G)$. 
- Create a new vertex $u$ and make it adjacent to every vertex of $\left\{y_{1}, \cdots, y_{n}\right\}$.

- Replicate $n$ copies $H_{1}, \cdots, H_{n}$ of $H$. For each $H_{i}$, arbitrarily choose two non-adjacent vertices $s$ and $t$ in its largest induced cycle, and identify $u$ with $s$, and $x_{i}$ with $t$.

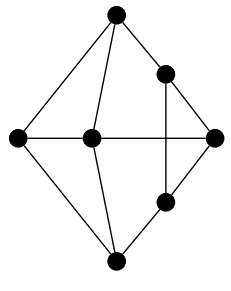

$H$

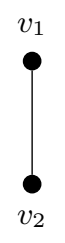

$G$

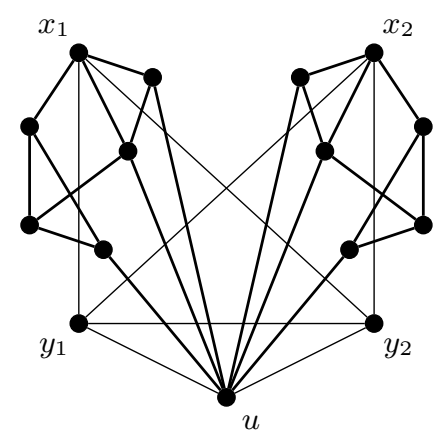

$G^{\prime}$

Fig. 4. An example of the reduction from Dominating Set to $H$-Free Contraction when $H$ is 3-connected and non-chordal

We claim that $G$ has a dominating $k$-set iff $G^{\prime}$ can be made into an $H$-free graph by contracting at most $k$ edges. Suppose that $T$ is a dominating $k$-set in $G$. We contract $k$ edges $\left\{u y_{i}: v_{i} \in T\right\}$ in $G^{\prime}$ to obtain a graph $G^{*}$, where $u$ is made adjacent to all vertices of $\left\{x_{1}, \cdots, x_{n}\right\}$. We show that $G^{*}$ is $H$-free.

Let $l(\geq 4)$ be the size of the largest induced cycle in $H$, and $t(\geq 1)$ be the number of different induced $l$-cycles in $H$. If $G^{*}$ contains an induced subgraph $H^{*}$ that is isomorphic to $H$, then $H^{*}$ also has $t$ different induced $C_{l}$. Observe that $G^{*}-\left(H_{1} \cup \cdots \cup H_{n}\right)$ is chordal, and each $H_{i}$ in $G^{*}$ contains at most $t-1$ induced $C_{l}$ because $u$ and $x_{i}$ is adjacent now. Thus there exists $p \neq q$ such that $V\left(H^{*}\right) \cap V\left(H_{p}\right) \neq \emptyset$ and $V\left(H^{*}\right) \cap V\left(H_{q}\right) \neq \emptyset$, which implies that $x_{p}, x_{q}, u \in V\left(H^{*}\right)$. However, removal of $u, x_{p}$ will disconnect $H^{*}$, contradicting to the fact that $H^{*}$ is 3 -connected. Therefore $G^{*}$ is an $H$-free graph.

Conversely, suppose that $G^{\prime}$ contains at most $k$ edges $F$ whose contraction results in a $H$-free graph. Similar to the proof in Theorem 4, there exists a set $F^{*}$ of at most $k$ edges such that contraction of $F^{*}$ destroys all induced copies $H_{1}, \cdots, H_{n}$ of $H$, and none edge of $F^{*}$ lies in these copies. We can use $F^{*}$ to obtain a dominating set of $G$ containing at most $\left|F^{*}\right| \leq k$ vertices.

Case 2. $H$ is chordal. The reduction for Case 1 does not work for 3-connected chordal $H$, because the constructed graph $G^{\prime}$ is a chordal graph. We will modify the reduction by subdividing the clique $\left\{y_{1}, \cdots, y_{n}\right\} \cup\{u\}$ and forcing contractions to occur in a specified set of edges.

Given an arbitrary instance $(G, k)$ of Dominating Set, we construct an instance $\left(G^{\prime}, 2 k\right)$ of $H$-Free Contraction in FPT time. Let $V(G)=\left\{v_{1}, \cdots, v_{n}\right\}$ and we construct graph $G^{\prime}$ as follows: 
- Create two independent sets: $\left\{x_{1}, \cdots, x_{n}\right\}$ and $\left\{y_{1}, \cdots, y_{n}\right\}$.

- Make $x_{i}$ adjacent to $y_{j}$ and mark this edge iff $i=j$ or $v_{i} v_{j} \in E(G)$.

- Create a new vertex $u$.

- For every pair of vertices $\{a, b\}$ in $\left\{y_{1}, \cdots, y_{n}\right\} \cup\{u\}$, create a degree-2 vertex $w_{a, b}$ which is made adjacent to $a$ and $b$. All these vertices constitute the subdivision of a $(n+1)$-vertex clique.

- Replicate $n$ copies $H_{1}, \cdots, H_{n}$ of $H$. For each $H_{i}$, arbitrarily choose two non-adjacent vertices $s$ and $t$, and identify $u$ with $s$, and $x_{i}$ with $t$. Mark all edges in $H_{i}$.

For every marked edge $e$, we will prevent it from being contracted. For this purpose, we need the following operation of attaching an expanded- $H$ to an edge $e$ of a graph: subdivide an edge $u v$ of a copy of $H$ by a vertex $w$, and identify edge $u w$ with edge $e$. Note that after this operation, the contraction of $e$ will generate a copy of $H$. We attach $2 k+1$ vertex-disjoint expanded- $H$ 's to $e$ to prevent $e$ from being contracted since contracting $e$ will generate $2 k+1$ induced copies of $H$ that cannot be destroyed by $2 k$ edge contractions. Thus we can only contract edges in $\left\{a w_{a, b}, b w_{a, b}: a, b \in\left\{y_{1}, \cdots, y_{n}\right\} \cup\{u\}\right\}$.

We claim that $G$ has a dominating $k$-set iff $G^{\prime}$ can be made into an $H$-free graph by at most $2 k$ edge contractions. Suppose that $T$ is a dominating $k$-set in $G$. We contract $2 k$ edges $\left\{u w_{u, y_{i}}, y_{i} w_{u, y_{i}}: v_{i} \in T\right\}$ of $G$ to obtain a graph $G^{*}$. Note that $u$ is identified with $\left\{y_{i}: v_{i} \in T\right\}$ in $G^{*}$ and therefore is adjacent to every vertex of $\left\{x_{1}, \cdots, x_{n}\right\}$, implying that $H_{1}, \cdots, H_{n}$ are destroyed. We show that $G^{*}$ is $H$-free.

Assume that $G^{*}$ contains an induced subgraph $H^{*}$ isomorphic to $H$. By the 3-connectivity of $H^{*}$, it is clear that $H^{*}$ is entirely inside the part of $G^{\prime}$ before attaching expanded- $H$ 's. Since the subgraph of $G^{*}$ induced by $\left\{x_{1}, \cdots, x_{n}\right\} \cup$ $\left\{y_{1}, \cdots, y_{n}\right\} \cup\{u\} \cup\left\{w_{a, b}: a, b \in\left\{y_{1}, \cdots, y_{n}\right\} \cup\{u\}\right\}$ is triangle-free, $H^{*}$ intersects $H_{i}$ for some $1 \leq i \leq n$. If $H^{*}$ contains a vertex outside $H_{i}$, then deleting $u$ and $x_{i}$ will disconnect this 3 -connected graph $H^{*}$, implying a contradiction. Thus, the vertex set $V\left(H^{*}\right)$ is exactly the set $V\left(H_{i}\right)$. However, the subgraph induced by $V\left(H_{i}\right)$ in $G^{*}$ has one more edge $u x_{i}$ than $H$, contradicting to the fact that $H^{*}$ is isomorphic to $H$. Therefore $G^{*}$ is an $H$-free graph.

Conversely, suppose that $G^{\prime}$ contains at most $2 k$ edges $F$ whose contraction results in an $H$-free graph. Note that subgraphs $H_{1}, \cdots, H_{n}$ in $G^{\prime}$ are destroyed by contracting $F$, which implies that $u$ is made adjacent to each $x_{i}$ in $G^{\prime} / F$. Let $S$ be a set of vertices in $\left\{y_{1}, \cdots, y_{n}\right\}$ that are finally in the same witness set with $u$ in $G^{\prime} / F$. We have $2|S| \leq|F|$ and $S$ dominates $\left\{x_{1}, \cdots, x_{n}\right\}$, implying that $G$ has a dominating set of at most $|S| \leq k$ vertices.

\section{Concluding Remarks}

We have studied $H$-Free Contraction problems in an attempt to obtain a dichotomy theorem for their parameterized complexity in terms of the structure of $H$, and we believe that techniques in the paper will be useful for further study 
of $\Pi$-Contraction problems. There are many natural and interesting problems about $H$-Free Contraction and $\Pi$-Contraction in general, and we will now discuss some open problems and propose some conjectures.

Unlike edge and vertex addition/deletions, edge contraction changes the structure of a graph less locally, and we feel that this nature makes edge contraction problems much more harder than edge and vertex modification problems. In general, we believe that $H$-FreE CONTRACTION is fixed-parameter intractable unless $H$ has a very special structure which limits the change.

Conjecture 7. For any fixed connected graph $H, H$-Free Contraction is $W$ [2]-hard unless $H$ is a complete graph or some graph with at most 5 vertices.

In light of the above conjecture, it will be important to determine whether $\mathrm{H}$ Free Contraction is FPT for small graphs, in particular for $H$ being $P_{4}$ and $K_{1,3}$. Note that $K_{1, t}$-Free Contraction is W[2]-hard for every fixed $t \geq 4$ [9].

Problem 8. Determine whether $P_{4}$-Free Contraction (or Cograph Contraction) and Claw-Free Contraction are FPT.

In connection with Conjecture 7, a confirmation of the following conjecture will be useful.

Conjecture 9. Let $H^{\prime}$ be an induced subgraph of $H$. Then $H$-Free ContraCTION is W[2]-hard whenever $H^{\prime}$-FreE CONTRACTION is.

For $P_{l}$-Free Contraction with even $l \geq 6$, we feel that it is useful to investigate how to prevent an edge from being contracted in order to settle the following conjecture.

Conjecture 10. For every fixed $l \geq 6, P_{l}$-Free Contraction is W[2]-hard.

Let $\mathcal{F}$ be a family of forbidden graphs. The $\mathcal{F}$-Free Contraction problem asks whether we can contract at most $k$ edges in $G$ to obtain a graph that is $H$-free for all $H \in \mathcal{F}$. Our work on $H$-Free Contraction may shed light on this general problem, and the following problem may serve as a good starting point.

Problem 11. Is it true that $\left\{H_{1}, H_{2}\right\}$-Free Contraction is W[2]-hard when both $H_{1}$-Free Contraction and $H_{2}$-Free Contraction are W[2]-hard, and FPT when both are FPT?

Finally, we believe that FPT algorithms for Clique Contraction and $K_{t^{-}}$ Free Contraction can be improved.

Problem 12. Design faster FPT algorithms for Clique Contraction and $K_{t}$-Free Contraction. 


\section{References}

1. Asano, T., Hirata, T.: Edge-deletion and edge-contraction problems. In: Proceedings of STOC 1982, pp. 245-254 (1982)

2. Asano, T., Hirata, T.: Edge-contraction problems. Journal of Computer and System Sciences 26(2), 197-208 (1983)

3. Cai, L.: Fixed-parameter tractability of graph modification problems for hereditary properties. Information Processing Letters 58(4), 171-176 (1996)

4. Chen, J., Kanj, I.A., Xia, G.: Improved upper bounds for vertex cover. Theoretical Computer Science 411(40-42), 3736-3756 (2010)

5. Dom, M., Lokshtanov, D., Saurabh, S.: Incompressibility through colors and IDs. In: Albers, S., Marchetti-Spaccamela, A., Matias, Y., Nikoletseas, S., Thomas, W. (eds.) ICALP 2009, Part I. LNCS, vol. 5555, pp. 378-389. Springer, Heidelberg (2009)

6. Eppstein, D.: Finding large clique minors is hard. Journal of Graph Algorithms and Applications 13(2), 197-204 (2009)

7. Golovach, P.A., Kamiński, M., Paulusma, D., Thilikos, D.M.: Increasing the minimum degree of a graph by contractions. Theoretical Computer Science 481, 74-84 (2013)

8. Golovach, P.A., van't Hof, P., Paulusma, D.: Obtaining planarity by contracting few edges. Theoretical Computer Science 476, 38-46 (2013)

9. Guo, C.: Parameterized Complexity of Graph Contraction Problems. PhD Thesis, The Chinese University of Hong Kong, Hong Kong S.A.R, China (2013), http://www.cse.cuhk.edu.hk/ cwguo/PhdThesis.pdf

10. Heggernes, P., van 't Hof, P., Lévêque, B., Lokshtanov, D., Paul, C.: Contracting graphs to paths and trees. In: Marx, D., Rossmanith, P. (eds.) IPEC 2011. LNCS, vol. 7112, pp. 55-66. Springer, Heidelberg (2012)

11. Heggernes, P., van't Hof, P., Lokshtanov, D., Paul, C.: Obtaining a bipartite graph by contracting few edges. In: Supratik, C., Amit, K. (eds.) FSTTCS 2011. LIPIcs, vol. 13, pp. 217-228. Leibniz-Zentrum für Informatik, Schloss Dagstuhl (2011)

12. Kaplan, H., Shamir, R., Tarjan, R.: Tractability of parameterized completion problems on chordal, strongly chordal, and proper interval graphs. SIAM Journal on Computing 28(5), 1906-1922 (1999)

13. Marx, D.: Chordal deletion is fixed-parameter tractable. Algorithmica 57(4), 747-768 (2010)

14. Robertson, N., Seymour, P.D.: Graph minors. XIII. The disjoint paths problem. Journal of Combinatorial Theory, Series B 63(1), 65-110 (1995)

15. Watanabe, T., Ae, T., Nakamura, A.: On the removal of forbidden graphs by edge-deletion or by edge-contraction. Discrete Applied Mathematics 3(2), 151-153 (1981) 